\title{
Comparison of Multiple Steroid Concentrations in Serum and Dried Blood Spots throughout the Day of Patients with Congenital Adrenal Hyperplasia
}

\author{
Kyriakie Sarafoglou ${ }^{a} \quad J o h n ~ H$. Himes $^{b} \quad$ Jean M. Lacey ${ }^{c} \quad$ Brian C. Netzel ${ }^{c}$ \\ Ravinder J.Singh ${ }^{c}$ Dietrich Matern ${ }^{c}$ \\ ${ }^{a}$ Department of Pediatrics and ${ }^{b}$ Division of Epidemiology and Community Health, School of Public Health, \\ University of Minnesota, Minneapolis, Minn., and ' ${ }^{\mathrm{D}}$ Department of Laboratory Medicine and Pathology, \\ Mayo Clinic College of Medicine, Rochester, Minn., USA
}

\section{Key Words \\ Congenital adrenal hyperplasia • \\ $17 \alpha$-hydroxyprogesterone $\cdot$ Tandem mass spectrometry • \\ Circadian rhythms of hormones $\cdot$ Glucocorticoid therapy}

\begin{abstract}
Background/Aim: Periodic measurement of plasma concentrations of cortisol precursors on a clinic visit may be of limited value in patients with congenital adrenal hyperplasia because it does not reflect a patient's circadian patterns of adrenal steroid secretion. Steroid profiling in dried blood spots (DBS) may allow for more frequent and sensitive monitoring. Methods: We compared the agreement between $17 \alpha$-hydroxyprogesterone (17-OHP) and androstenedione (D4A) levels determined from DBS samples and concurrently collected serum samples. Blood was drawn from 9 congenital adrenal hyperplasia patients every 4 h over a 24 -hour period. Serum and DBS steroid levels were measured by liquid chromatography tandem mass spectrometry. Results: DBS determinations of 17-OHP overestimated corresponding serum levels (mean difference $1.67 \mathrm{ng} / \mathrm{ml}$ ), and underestimated D4A serum levels (mean difference $0.84 \mathrm{ng} / \mathrm{ml}$ ). However, the DBS assay yielded excellent agreement (97\%) with serum 17-OHP, but did considerably poorer for D4A (31\%). Conclu-
\end{abstract}

sions: Our results indicate an excellent agreement between DBS and serum 17-OHP measurements to identify the peaks and troughs associated with an individual's circadian pattern. Larger-scale studies are required to evaluate the utility of DBS for home monitoring and to determine if more frequent monitoring leads to improved clinical outcomes.

Copyright $\odot 2010$ S. Karger AG, Basel

\section{Introduction}

Optimal control of adrenal function in congenital adrenal hyperplasia $(\mathrm{CAH})$ due to $21 \alpha$-hydroxylase deficiency (21-OHD) is of paramount importance during childhood and adolescence, where excess adrenal androgens manifest as growth acceleration, advanced bone age, short stature, early puberty, excess facial and body hair, acne, and (in females) irregular menstrual periods [1-3]. Current replacement treatment (glucocorticoids) does not precisely replicate the circadian rhythm of cortisol secretion because it lacks the close temporal relationship to adrenocorticotropin (ACTH) pulses, and increased adrenal activity will continue despite treatment. Increasing treatment doses to achieve satisfactory androgen suppression exposes the patient to the negative effects gluco-

\section{KARGER}

() 2010 S. Karger AG, Basel

Fax +4161306 1234

E-Mail karger@karger.ch

www.karger.com
Accessible online at: www.karger.com/hrp
Kyriakie Sarafoglou, MD

University of Minnesota, MMC 8404 13-124 PWB

516 Delaware St. SE

Minneapolis, MN 55455 (USA)

Tel. +1 612624 5409, Fax +1 612626 5262, E-Mail saraf010@umn.edu 
Table 1. Characterization and medication of study subjects

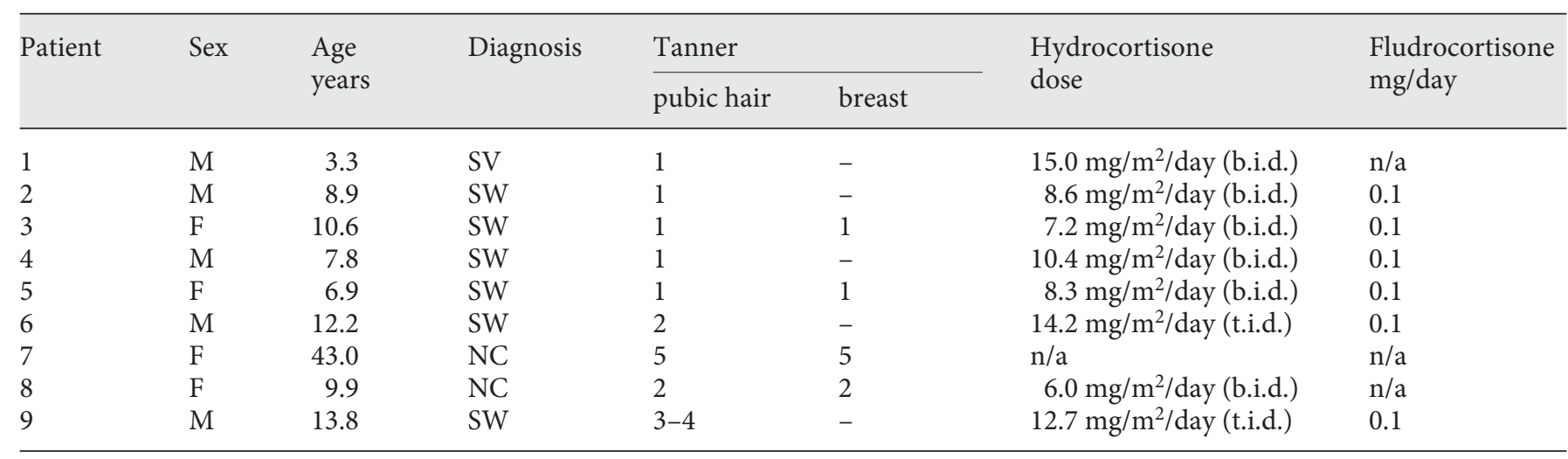

corticoids have on growth, explaining why some 'wellcontrolled' CAH patients may experience early puberty, compromised final height, polycystic ovarian disease, and male infertility $[4,5]$.

$17 \alpha$-hydroxyprogesterone (17-OHP), androstenedione (D4A) and/or testosterone are precursors used to assess adrenal control, and they are typically measured in childhood every 3-6 months during a clinic visit; renin and electrolytes are additionally monitored in patients with the salt-wasting (SW)-CAH variant. However, a single serum measurement has been shown to be of limited use in assessing the true metabolic status of a $\mathrm{CAH}$ patient between follow-up appointments $[6,7]$ because it does not indicate the patient's individual circadian pattern of adrenal cortisol secretion over the course of the day. More frequent monitoring, including multiple measurements within a 24 -hour period, so that that treatment can be adjusted more closely to a patient's circadian rhythm of adrenal secretion, may improve long-term outcomes. Other methods of monitoring, 24-hour urinary 17-ketosteroids for example, have fallen out of favor because of the difficulty in collecting 24-hour urine in infants and children every 3-4 months and may require bladder catheterization to perform. In addition, it has been established that serum 17-OHP levels above $10 \mathrm{ng} /$ $\mathrm{ml}$ consistently correlate with abnormal levels of urinary 17-ketosteroids and adrenal androgens [8].

Immunoassay measurements of 17-OHP in dried blood spots (DBS) on filter paper obtained from a skin prick is currently used by newborn screening programs throughout the USA and Europe. More recently, improved methods making use of liquid chromatography tandem mass spectrometry (LC-MS/MS) have become available; they allow measurement of 17-OHP and additional steroids in DBS and are used for 2nd-tier newborn screening [9-11]. Because of the minimal volume of blood needed and the ease of obtaining and shipping (at ambient temperature) the samples, it may be feasible to better tailor cortisol replacement therapy of $\mathrm{CAH}$ patients by regular measurement of adrenal steroids in capillary blood collected at home via skin prick. Our study examines whether there is sufficient agreement between multiple measurements of adrenal steroid concentrations (17OHP and D4A) in DBS and serum collected simultaneously over a 24-hour period, and if corresponding clinical decisions would differ between the two methods.

\section{Materials and Methods}

\section{Subjects and Research Design}

A group of 9 patients (4 females and 5 males) with CAH due to 21-OHD as confirmed by biochemical and genetic analyses were admitted to the General Clinical Research Center at the University of Minnesota Medical Center for $24 \mathrm{~h}$. Six patients had SW-CAH, one had simple-virilizing (SV)-CAH and 2 patients had nonclassical (NC)-CAH. The age range of the patients was 3.3-43 years (mean: 9.36 years). Six out of 9 patients were prepubertal, one was in puberty (Tanner stage 3-4 for pubic hair and testicular volume), one had his puberty suppressed by monthly injections of leuprolide (Lupron) and one was an adult female. Patients with SW-CAH, SV-CAH and one with $\mathrm{NC}-\mathrm{CAH}$ were treated with hydrocortisone (Cortef) at a dose of $6-15 \mathrm{mg} / \mathrm{m}^{2} /$ day (table 1). Six patients were receiving hydrocortisone twice a day and 2 patients three times a day. All 6 patients with SW-CAH were on $0.1 \mathrm{mg}$ of fludrocortisone once daily. The adult female patient with $\mathrm{NC}-\mathrm{CAH}$ was not taking any medications.

The study was conducted under a protocol approved by the Institutional Review Board of the University of Minnesota Medi- 
Table 2. Categorical agreement between serum and DBS assessments of $17-\mathrm{OHP}^{1}$ and $\mathrm{D}^{4} \mathrm{~A}^{2}$ by time of day

\begin{tabular}{|c|c|c|c|c|}
\hline \multirow[t]{2}{*}{ Time } & \multicolumn{2}{|l|}{ 17-ОHP } & \multicolumn{2}{|l|}{$\mathrm{D} 4 \mathrm{~A}$} \\
\hline & $\begin{array}{l}\text { count } \\
\text { agreement }\end{array}$ & $\kappa^{3}$ & $\begin{array}{l}\text { count } \\
\text { agreement }\end{array}$ & $\kappa^{3}$ \\
\hline 8 a.m. & $8 / 8$ & 1.00 & $4 / 9$ & $0.015^{4}$ \\
\hline 12 p.m. & $9 / 9$ & 1.00 & $5 / 9$ & 0.294 \\
\hline 4 p.m. & $9 / 9$ & 1.00 & $6 / 9$ & 0.491 \\
\hline 8 p.m. & $9 / 9$ & 1.00 & $5 / 9$ & 0.368 \\
\hline 12 a.m. & $9 / 9$ & 1.00 & $6 / 9$ & $0.298^{4}$ \\
\hline 4 a.m. & $9 / 9$ & 1.00 & $7 / 9$ & $0.562^{4}$ \\
\hline 8 a.m. & $7 / 8$ & 0.784 & $4 / 9$ & 0.167 \\
\hline Total/average & $60 / 61$ & 0.969 & $37 / 63$ & 0.314 \\
\hline
\end{tabular}

${ }^{1}$ 17-OHP categories (ng/ml): $<10 ; 10.0-19.99 ; 20.0-34.99 ; \geq 35$.

${ }^{2} \mathrm{D} 4 \mathrm{~A}$ categories for low, normal, and high according to Soldin et al. [15].

\begin{tabular}{llllll}
\hline \multicolumn{1}{c}{ Tanner stages: } & I & II & III & IV & V \\
\hline Range for males, ng/dl & $<15-50$ & $31-65$ & $50-100$ & $48-140$ & $65-210$ \\
Range for females, ng/dl & $<15-50$ & $42-100$ & $80-190$ & $77-225$ & $80-240$
\end{tabular}

\footnotetext{
${ }^{3}$ Kappa coefficient of chance-adjusted agreement.

${ }^{4}$ Kappa coefficient approximated by square of Spearman correlation because of missing cells.
}

\section{Statistical Analysis}

The distributions of the hormone concentrations were substantially skewed toward higher values, but they approached normality by transforming observed values to logarithms (base 10). Geometric means, standard errors and differences between serum and filter-paper hormone concentrations were obtained using general linear models, nesting the multiple measures throughout the day within each subject by using the subject as a random effect. This approach accounted for the lack of independence of hormone concentrations within each subject. Paired $t$ tests were calculated using the transformed variables to satisfy the assumptions of normality. Estimates of the means and differences were then back-transformed into the original scale ( $\mathrm{ng} / \mathrm{ml})$. Intraclass correlation coefficients estimating the proportion of variation in the serum estimates accounted for by the estimates from DBS were calculated using two-way random effects models. In this situation, intraclass correlations are used because they account for both random and systematic errors.

To determine if clinical decisions based on the adrenal steroid concentrations from DBS might differ from corresponding decisions from serum assays taken at the same times, the agreement between the two approaches was tabulated with respect to cutoffs designating meaningful categories of the respective hormone levels. For $17-\mathrm{OHP}$, the categories $(\mathrm{ng} / \mathrm{ml})$ were: $<10 ; 10.0-19.99$; 20.0-34.99; $\geq 35$. For D4A, categories were assigned for low, normal, and high based on gender and Tanner stage of individual subjects, according to established reference ranges [15] listed in the legend of table 2. Kappa coefficients expressing chance-adjusted agreement were calculated for each time sample. In cases where there were empty cells in the cross-tabulations, kappa was approximated by the value of the corresponding Spearman rank correlation squared.

\section{Results}

and parents/guardians.

Subjects used their own medications and followed their normal schedule with the exception of the first morning dose which was held until 8 a.m. blood samples were obtained. The following morning's dose of medications was also withheld until the last blood sample was drawn. Every $4 \mathrm{~h}$ for $24 \mathrm{~h}$, blood samples were taken, $2 \mathrm{ml}$ for serum and approximately $100 \mu \mathrm{l}$ to prepare DBS by spotting whole blood on filter paper (Whatman Protein Saver 903). The accuracy of measurements of 17-OHP and D4A concentrations in DBS was determined by comparisons to concentrations of the same analytes in the corresponding serum samples.

\section{Hormone Assays}

Serum steroids were determined using methods applied in the authors' clinical laboratory. Briefly, deuterated stable isotope internal standards were added to $100 \mu \mathrm{l}$ serum. Each sample was then exposed to solid phase extraction on Strata X 30-mg cartridges, and eluted with methanol. Extracts were then dried under a nitrogen stream, reconstituted to $100 \mu \mathrm{l}$ in mobile phase and analyzed by LC-MS/MS using multiple reaction monitoring in electrospray ionization mode [12]. Analysis of DBS was performed as previously described $[9,13]$ with modifications to include quantification of the same steroids as in serum following extraction by turbulent flow chromatography using isotopically labeled internal standards [14].

Comparison of Steroid Concentrations in Serum and Dried Blood Spots
Table 3 shows that DBS determinations of 17-OHP overestimated corresponding levels measured in serum by a mean of $1.67 \mathrm{ng} / \mathrm{ml}$, and DBS assays of D4A underestimated serum assessments by a mean of $0.84 \mathrm{ng} / \mathrm{ml}$. Both of the mean differences are statistically significant $(p<0.05)$. The intraclass correlations between the methods are moderately high relative to the ideal of 1.0, with that for 17-OHP (0.884) being greater than that for D4A (0.724). Inspection of the data patterns indicated that the differences between DBS and serum determinations were largest at the highest serum concentrations, especially for D4A (data not shown).

Accordingly, it is clinically important to ask if the observed average biases with DBS would yield different clinical conclusions in identifying meaningful categories of adrenal steroid concentrations compared with serum determinations over a 24 -hour period. Table 2 presents the correspondence counts and kappa coefficients of chance-adjusted agreement relative to identifying cate- 
Table 3. Geometric means, differences, and intraclass correlations for serum and DBS assessments of 17-OHP and D4A

\begin{tabular}{|c|c|c|c|c|c|c|c|c|c|}
\hline Variable & \multicolumn{2}{|l|}{ Serum } & \multicolumn{2}{|l|}{ DBS } & \multicolumn{2}{|c|}{ Difference } & t statistic & $\mathrm{p}$ & Intraclass $\mathrm{R}$ \\
\hline 17-OHP, ng/ml & 3.51 & & 6.44 & & 1.67 & & & & \\
\hline $\log \mathrm{D} 4 \mathrm{~A}$ & -0.399 & 0.045 & -0.473 & 0.026 & -0.074 & 0.028 & 2.64 & $<0.05$ & 0.724 \\
\hline $\mathrm{D} 4 \mathrm{~A}, \mathrm{ng} / \mathrm{ml}$ & 0.40 & & 0.34 & & -0.84 & & & & \\
\hline
\end{tabular}

gories of 17-OHP and D4A by time of blood collection. Even though DBS measurements tend to overestimate actual 17-OHP concentrations, DBS results, in this limited study, were in excellent agreement with serum results using the specified categories, with a total absolute agreement of $97 \%$. DBS assessments of D4A did considerably worse in correctly identifying low, normal and elevated levels, with the total absolute agreement being only $59 \%$.

\section{Discussion}

Successful treatment of patients with CAH 21-OHD, especially of growing children, requires a fine balance between achieving androgen suppression without exposing the patient to glucocorticoid excess. Although several dosage schedules of hydrocortisone preparations have been recommended to attain this balance, dosage and scheduling regimens are difficult to optimize among patients due to varying medication clearance and the peaks and troughs of adrenal steroid secretion during the day. Healthy individuals with normal sleep patterns have a distinct circadian rhythm which consists of: (1) undetectable cortisol and ACTH levels at midnight; (2) a sharp rise during the last hours of sleep reaching a peak at 4-6 a.m.; (3) a steady decline throughout the rest of the waking day, with superimposition of variable peaks of cortisol secretion triggered by stress, meals and exercise [16]. Ideal glucocorticoid replacement therapy should mirror the circadian patterns as closely as possible, including the early morning rise in the ACTH and adrenal androgens. To tailor the individual patient's hydrocortisone regimen, multiple measurements of adrenal steroids over a 24hour period may be useful but only if they were easy to obtain.

To determine the feasibility of DBS as a convenient sample, our study assessed the agreement of 17-OHP and
D4A measurements in DBS and serum taken at the same time over the course of $24 \mathrm{~h}$, and if the same clinical decisions would be made based on the results. For 17-OHP (ng/ml) we assigned four categories: <10; 10.0-19.99; 20.0-34.99; $\geq 35.0$. Hughes and Winter [8] have shown that values $<10 \mathrm{ng} / \mathrm{ml}$ are not associated with significant elevation of adrenal androgens; $10-19.99$ indicates a mild elevation of $17-\mathrm{OHP} ; 20-34.99$ signals a mild to moderate elevation, and $\geq 35$ indicates a moderate to high elevation. Though DBS overestimated corresponding 17-OHP serum levels by a mean of $1.67 \mathrm{ng} / \mathrm{ml}$, we had categorical agreement in 60 out of 61 pairs, suggesting that DBS could be used to identify meaningful aspects of an individual's circadian pattern over a 24 -hour period and provide a fuller picture of disease control. In the one instance where categorical agreement between serum and DBS 17OHP values was not found $(28.6$ and $7.44 \mathrm{ng} / \mathrm{ml}$, respectively), the difference was substantial and reproducible by repeat analysis of both samples. The next two largest differences were seen at the two highest serum 17-OHP levels of 192 and $82 \mathrm{ng} / \mathrm{ml}$ (DBS 254 and $57.8 \mathrm{ng} / \mathrm{ml}$, respectively), though both levels fell into the same agreement category.

The DBS assessment of D4A underestimated corresponding serum levels by a mean of $0.84 \mathrm{ng} / \mathrm{ml}$ and was in poor agreement $(59 \%)$ with correctly identifying low, normal and elevated levels defined by Soldin et al. [15]. However, we did observe that the fluctuation of D4A generally followed a similar secretion pattern as 17-OHP (fig. 1,2), which challenges previous reports that $\mathrm{D} 4 \mathrm{~A}$ is a more reliable marker to monitor control of CAH 21OHD because it is less affected by circadian rhythm and is less influenced by individual steroid doses than 17OHP levels $[17,18]$. The nonfluctuation of D4A during the 24-hour observations in these studies was probably the result of oversuppression because, at the time they were conducted (1970s and 80s), standard glucocorticoid doses given to patients were significantly higher. 

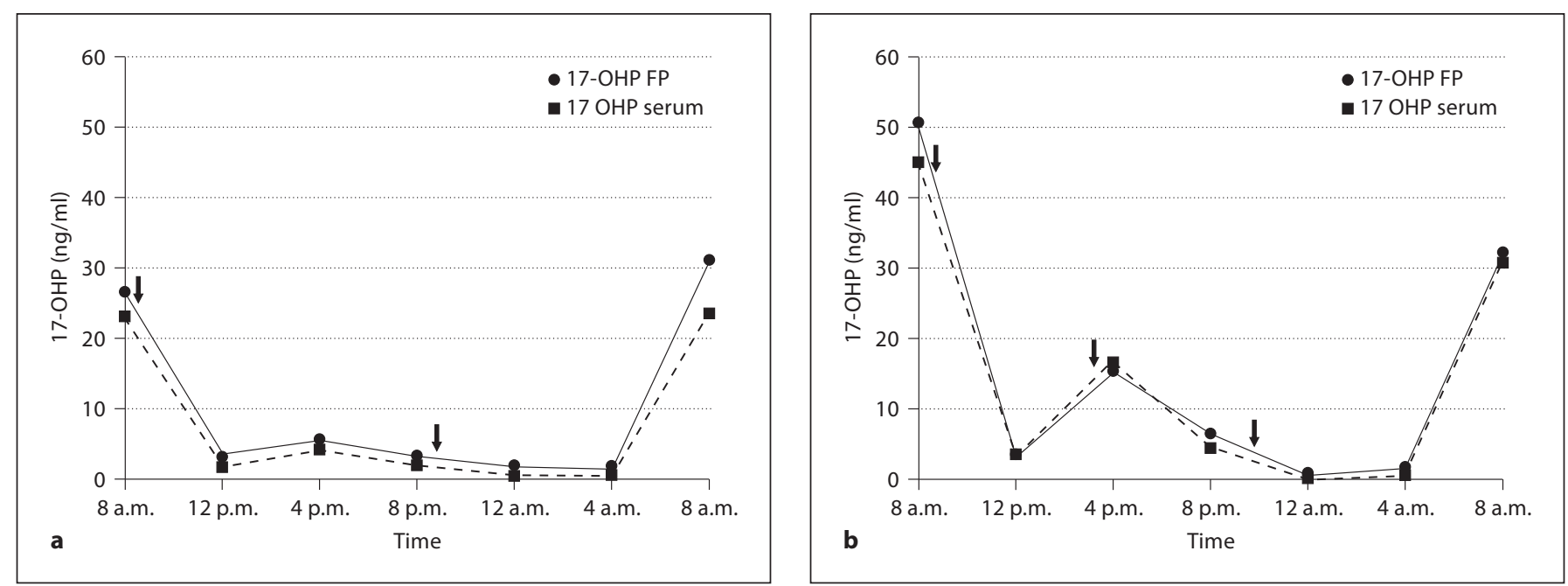

Fig. 1. Serum and DBS measurements of 17-OHP over a 24-hour period in patient 3 (a) and 6 (b). Black arrows indicate the time the patient took hydrocortisone. Both patients took fludrocortisone with morning hydrocortisone dose (approximately 8 a.m.). FP $=$ Filter paper.
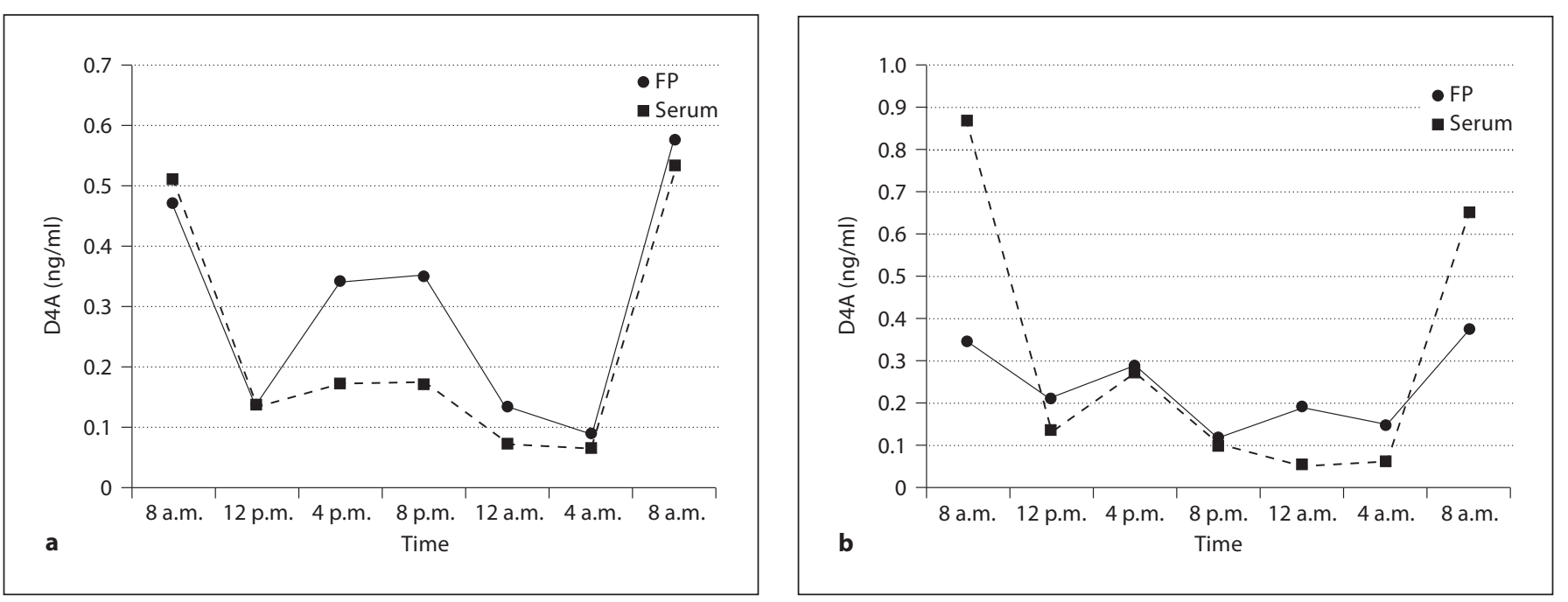

Fig. 2. Serum and DBS measurements of D4A over a 24-hour period in patient 3 (a) and 6 (b).

The reason for the discrepancy between steroids determined in DBS and serum samples collected at the same time is uncertain, in particular for those samples that may represent outliers for unknown reasons. Incomplete extraction of steroids from DBS is unlikely given that 17-OHP was generally higher when measured in DBS. Participation in the Centers for Disease Control and Prevention's Newborn Screening Quality Assurance program for steroid analysis by LC-MS/MS in DBS reveals similar performance of the assay used here to those of other laboratories [19]. Nevertheless, steroid analysis in DBS cannot simply replace serum analysis until its robustness has been documented in larger comparative studies and DBS-specific reference ranges have been established. 
A potential advantage of more frequent measurements of 17-OHP in DBS over a 24 -hour period versus single serum measurements was seen in 2 subjects, both of whom had elevated $17-\mathrm{OHP}$ values $(>10 \mathrm{ng} / \mathrm{ml})$ in morning measurements (8 a.m.), indicating a nocturnal surge of adrenal steroids (fig. 1). Subject 3 maintained normal afternoon and evening 17-OHP levels on twice-a-day hydrocortisone and subject 6 had elevated midday 17-OHP levels on a 3-times-a-day hydrocortisone regimen (see table 1). If the hydrocortisone dose and schedule of these patients were based on only the morning 17-OHP level (8 a.m.), either the morning or evening dose of hydrocortisone would be increased in these patients depending on physician's preference [20-22]. However, with knowledge of the 17-OHP concentrations at different times of the day, administration of the same hydrocortisone dose later in the evening or an increase in the evening dose of patient 3 could be used to prevent the nocturnal surge. As morning levels are higher in patient 6 , and the patient displayed a midday 17-OHP surge, possible changes to the dosing schedule might include giving the midday hydrocortisone earlier and the evening dose later (if the physician preferred not to raise the hydrocortisone dose) or slightly increasing the midday and evening doses or some variation in between.
Monitoring of adrenal steroids using DBS may provide a practical means of assessing the efficacy of replacement therapy over the course of the day. Preparing DBS at home by a finger stick may be acceptable to many patients and would allow for more frequent assessments of their adrenal steroid control, which is of paramount importance in childhood because glucocorticoids have a doseand age-dependent negative effect on linear growth [2325]. However, larger studies are needed to further investigate the observed disagreement between DBS and serum steroid levels, to determine specific DBS reference ranges for both $17-\mathrm{OHP}$ and $\mathrm{D} 4 \mathrm{~A}$, to evaluate the feasibility of home monitoring, and to prospectively compare the long-term clinical outcome between patients with traditional, cursory measurement of steroids in serum and those obtaining several DBS at home according to a standardized schedule.

\section{Acknowledgements}

This work was supported, in part, by M01-RR00400 National Center for Research Resources, National Institutes of Health; R03 HD046531 National Institute of Child Health and Human Development.

\section{References}

1 Sarafoglou K, Harrington KD, Bockting WO: Congenital adrenal hyperplasia; in Sarafoglou K, Hoffmann GF, Roth KS (eds): Pediatric Endocrinology and Inborn Errors of Metabolism. New York, McGraw-Hill, 2009, pp 385-410.

-2 Van der Kamp HJ, Otten BJ, Buitenweg N, De Muinck Keizer-Schrama SM, Oostdijk W, Jnsen M, Delemarre-de Waal HA, Vulsma T, Wit JM: Longitudinal analysis of growth and puberty in 21-hydroxylase deficiency patients. Arch Dis Child 2002;87:139-144.

3 Hargitai G, Solyom J, Battelino T, Lebl J, Pribilincová Z, Hauspie R, Kovács J, Waldhauser F, Frisch H: Growth patterns and final height in congenital adrenal hyperplasia due to classical 21-hydroxylase deficiency. Results of a multicenter study. Horm Res 2001;55:161-171.

-4 Urban MD, Lee PA, Migeon CJ: Adult height and fertility in men with congenital virilizing adrenal hyperplasia. N Engl J Med 1978; 299:1392-1396.

5 Jaaskelainen J, Tiitinen A, Voutilainen R: Sexual function and fertility in adult females and males with congenital adrenal hyperplasia. Horm Res 2001;56:73-80.
6 Frisch H, Parth K, Schober E, Swoboda W: Circadian patterns of plasma cortisol, 17-hydroxyprogesterone, and testosterone in congenital adrenal hyperplasia. Arch Dis Child 1981;56:208-213.

7 Atherden SM, Barnes ND, Grant DB: Circadian variation in plasma 17-hydroxyprogesterone in patients with congenital adrenal hyperplasia. Arch Dis Child 1972;47:602604.

$\$ 8$ Hughes IA, Winter JSD: The relationships between serum concentrations of $17-\mathrm{OH}-$ progesterone and other serum and urinary steroids in patients with congenital adrenal hyperplasia. J Clin Endocrinol Metab 1978; 46:98-104.

-9 Lacey JM, Minutti CZ, Magera MJ, Tauscher AL, Casetta B, McCann M, Lymp J, Hahn SH, Rinaldo P, Matern D: Improved specificity of newborn screening for congenital adrenal hyperplasia by second-tier steroid profiling using tandem mass spectrometry. Clin Chem 2004;50:621-625.
10 Janzen N, Peter M, Sander S, Steuerwald U, Terhardt M, Holtkamp U, Sander J: Newborn Screening for Congenital Adrenal Hyperplasia: additional steroid profile using liquid chromatography-tandem mass spectrometry. J Clin Endocrinol Metab 2007;92:25812589.

-11 Schwarz E, Liu A, Randall H, Haslip C, Keune F, Murray M, Longo N, Pasquali M: Use of steroid profiling by UPLC-MS/MS as a second tier test in newborn screening for congenital adrenal hyperplasia: the Utah experience. Pediatr Res 2009;66:230-235.

12 Kao PC, Machacek DA, Magera MJ, Lacey JM, Rinaldo P: Diagnosis of adrenal cortical dysfunction by liquid chromatography-tandem mass spectrometry. Ann Clin Lab Sci 2001;31:199-204.

13 Minutti CZ, Lacey JM, Magera MJ, Hahn SH, McCann M, Schulze A, Cheillan D, Dorche C, Chace DH, Lymp JF, Zimmerman D, Rinaldo P, Matern D: Steroid profiling by tandem mass spectrometry improves the positive predictive value of newborn screening for congenital adrenal hyperplasia. J Clin Endocrinol Metab 2004;89:3687-3693. 
14 Lacey JM, Lorey F, Tortorelli S, Gavrilov D, Rinaldo P, Matern D: Newborn screening for congenital adrenal hyperplasia by turbulent flow chromatography and LC-MS/MS; in Proc Newborn Screen Genet Test Symp, May 2007, Minneapolis. Silver Spring, Association of Public Health Laboratories, 2007, p 39.

15 Soldin SJ, Brugnara C, Wong EC (eds): Pediatric Reference Ranges, ed 4. Washington, AACC Press, 2003, pp 32-34.

16 Krieger DT, Allen W, Rizzo F, Krieger HP: Characterization of the normal temporal pattern of plasma corticosteroid levels. J Clin Endocrinol Metab 1971;32:266-284.

17 Egan SM, Betts P, Thomson S, Wallace AM, Wood PJ: A bloodspot androstenedione assay suitable for home-monitoring of steroid replacement therapy in congenital adrenal hyperplasia. Ann Clin Biochem 1989;26: 262-267.
18 Lee PA, Urban MD, Gutai JP, Migeon CJ: Plasma progesterone, 17-OHP, androstenedione and testosterone in prepubertal, pubertal and adult subjects with congenital vir ilizing adrenal hyperplasia as indicators of adrenal suppression. Horm Res 1980;13: 347-357.

19 De Jesus VR, Mei JV, Bell CJ, Hannon WH: Improving and assuring newborn screening laboratory quality worldwide: 30 -year experience at the centers for disease control and prevention. Semin Perinatol 2010;34:125133.

20 Charmandari E, Matthews DR, Johnston A, Brook CGD, Hindmarsh PC: Serum cortisol and 17-hydroxyprogesterone. Interrelation in classic 21-hydroxylase deficiency: Is current replacement therapy satisfactory? J Clin Endocrinol Metab 2001;86:4679-4685.

21 Ross RJ, Rostami-Hodjegan A: Timing and type of glucocorticoid replacement in adult congenital adrenal hyperplasia. Horm Res 2005; $64: 67-70$
22 Hughes IA: Management of congenital adrenal hyperplasia. Arch Dis Child 1988;63: 1399-1404.

23 Jaaskelainen J, Voutilainen R: Growth of patients with 21-hydroxylase deficiency: an analysis of the factors influencing adult height. Pediatr Res 1997;41:30-33.

24 Manoli I, Kanaka-Gantenbein Ch, Voutetakis A, Maniati-Christidi M, Dacou-Voutetakis C: Early growth, pubertal development, body mass index and final height of patients with congenital adrenal hyperplasia: factors influencing the outcome. Clin Endocrinol (Oxf) 2002;57:669-676.

25 Stikkelbroeck NM, Van't Hof-Grootenboer BA, Hermus AR, Otten BJ, Van't Hof MA: Growth inhibition by glucocorticoid treatment in salt-wasting 21-hydroxylase deficiency: in early infancy and (pre) puberty. J Clin Endocrinol Metab 2003;88:3525-3530. 